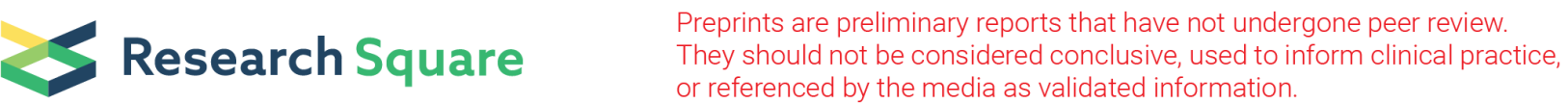

\section{Analysis of Spatial-Temporal Distribution of Respiratory Infectious Diseases in Shandong Province, China During 2005-2014}

\section{Xiaomei Li}

Shandong First Medical University - Tai'an Campus

\section{Dongzhen Chen}

Shandong First Medical University - Tai'an Campus

\section{Yan Zhang}

The Affiliated Hospital of Guizhou Medical University

\section{Xiaojia Xue}

Qingdao Municipal Center for Disease Control and Prevention

\section{Xuewen Li}

Shandong University

\section{Shengyang Zhang}

Shandong Center for Disease Control and Prevention

\section{Meng Chen}

Jining Centers for Disease Control and Prevention

Xuena Liu

Shandong First Medical University - Tai'an Campus

Guoyong Ding ( $\nabla$ dgy-153@163.com )

Shandong First Medical University - Tai'an Campus

\section{Research Article}

Keywords: Respiratory infectious diseases, Spatial-temporal distribution, Spatial autocorrelation, Spatialtemporal clustering

Posted Date: September 18th, 2020

DOl: https://doi.org/10.21203/rs.3.rs-77345/v1

License: (c) (1) This work is licensed under a Creative Commons Attribution 4.0 International License.

Read Full License 


\section{Abstract}

Background: Little comprehensive information on overall epidemic trend of respiratory infectious diseases is available in Shandong Province, China. This study aimed to determine the spatiotemporal distribution and epidemic characteristics of respiratory infectious diseases.

Methods: Time series was firstly performed to describe the temporal distribution feature of respiratory infectious diseases during 2005-2014 in Shandong Province. GIS Natural Breaks (Jenks) was applied to divide the average annual incidence of respiratory infectious diseases into five grades. Spatial empirical Bayesian smoothed risk maps and excess risk maps were further used to investigate spatial patterns of respiratory infectious diseases. Global and local Moran's / statistics were used to measure the spatial autocorrelation. Spatial-temporal scanning was used to detect spatiotemporal clusters and identify highrisk locations.

Results: A total of 537,506 cases of respiratory infectious diseases were reported in Shandong province during 2005-2014. The morbidity of respiratory infectious diseases had obvious seasonality with high morbidity in winter and spring. Local Moran's / analysis showed that there were 5, 23, 24, 4, 20, 8, 14, 10 and 7 high-risk counties determined for influenza A (H1N1), measles, tuberculosis, meningococcal meningitis, pertussis, scarlet fever, influenza, mumps and rubella, respectively. The spatial-temporal clustering analysis determined that the most likely cluster of influenza $A(H 1 N 1)$, measles, tuberculosis, meningococcal meningitis, pertussis, scarlet fever, influenza, mumps and rubella included $74,66,58,56$, 22, 64, 2, 75 and 56 counties, and the time frame was November 2009, March 2008, January 2007, February 2005, July 2007, December 2011, November 2009, June 2012 and May 2005, respectively.

Conclusions: There were obvious spatiotemporal clusters of respiratory infectious diseases in Shandong during 2005-2014. More attention should be paid to the epidemiological and spatiotemporal characteristics of respiratory infectious diseases to establish new strategies for its control.

\section{Background}

Although the incidence of infectious diseases has been effectively controlled, infectious diseases are still a major global public health problem. At the global level, infectious diseases amount to an estimated $18 \%$ of the total disability adjusted life years (DALYs) of the Global Burden of Disease (GBD) in 2016[1]. At present, infectious diseases still represent a significant public health problem in China, with over 10 million cases (incidence rate was 733.57 per 100,000) reported in 2019[2]. There are currently 40 notifiable infectious diseases in China (including coronavirus disease 2019), which are classified as intestinal infectious diseases, respiratory infectious diseases, vector-borne diseases, blood and sexually transmitted diseases, and neonatal tetanus according to the transmission of infectious diseases[3]. Respiratory infectious diseases resulting from bacterial or viral pathogens such as mycobacterium tuberculosis, streptococcus pneumoniae, respiratory syncytial virus (RSV) or influenza virus are major global public health concerns. For example, pandemic of coronavirus disease 2019 (one of respiratory 
infectious diseases) led to 12.77 million cases and 0.57 million deaths worldwide in seven months[4]. The outbreak of respiratory infectious diseases may lead to a looming public health crisis. Being one of the provinces with high incidence rates of respiratory infections diseases in China, Shandong Province had an annual incidence rate of about 44.12 per 100,1000 in the total population in recent years[5].

The spread of disease can vary significantly from place to place and from time to time for diverse causes[6]. Describing the epidemiological distribution of respiratory infectious diseases is important for the monitoring and prevention of respiratory infectious diseases and acts as the basis for further research. Geographic information systems (GIS), together with cluster analysis tools, establish an approach to delimiting research on specific population groups and/ or areas and make it effective, helping to explore epidemic hot spots of respiratory infectious diseases. Many studies have explored the distribution of spatial-temporal clusters in the epidemiology of a particular type of respiratory infectious diseases. For example, the spatial and temporal characteristics of influenza A (H7N9) was described in China using GIS method[7-9]. One study provided a quantitative description of the age-specific morbidity pandemic patterns of influenza A (H1N1) across administrative areas of Peru[10]. Several studies about the spatial-temporal distribution of tuberculosis such as Beijing[11], Yunnan[12, 13], Qinghai[14] and mainland of China[15] were analyzed in China. Based on GIS technology and spatial statistics, one study explored areas and periods at high risk of scarlet fever in China[16]. Another Chinese study was conducted to evaluate the significance of aggregation and determine the size of the range of hotspots at the county level in Guangxi[17]. These studies have shown the geographical and temporal heterogeneity of respiratory infectious diseases epidemic.

To our knowledge, only one study has systematically explored the epidemiologic trends and spatial changing patterns of respiratory infectious diseases, but which analyzed at the provincial level in China[18]. Therefore, cluster analysis of respiratory infectious diseases at a more precise level is urgently needed. With little research has been conducted in Shandong Province, China, the overall epidemic trend of respiratory infectious diseases remains unknown. This study aimed to detect the spatial-temporal clusters of respiratory infectious diseases at county-level of Shandong. Knowledge of spatial-temporal distribution of respiratory infectious diseases was crucial to understand the dynamic transmission of respiratory infectious diseases and to provide local evidence for prevention and control strategies of respiratory infectious diseases.

\section{Methods}

\section{Ethical Statement}

Disease surveillance data used in this study were obtained from the China Information System for Disease Control and Prevention with the approval by China CDC. All data are unidentified. The present study was reviewed by the research institutional review board of Shandong First Medical University, and it is found that utilization of disease surveillance did not require oversight by an ethics committee.

\section{Study area}


The study was conducted in Shandong Province (longitude $114^{\circ} 47.5^{\prime}-122^{\circ} 42.3^{\prime} \mathrm{E}$ and latitude $34^{\circ} 22.9^{\prime}-38^{\circ} 24.01^{\prime} \mathrm{N}$ ), which is located in the lower reaches of Yellow River with Bohai Sea and Yellow Sea in the East (See Supplementary Fig. 1, Additional File 1). By 2020, Shandong Province has 16 prefecturelevel cities (i.e., Jinan, Qingdao, Zibo, Zaozhuang, Dongying, Yantai, Weifang, Jining, Taian, Weihai, Rizhao, Binzhou, Liaocheng, Linyi, and Heze), 57 municipal districts, 27 county-level cities, 53 counties and 137 county-level administrative regions in total. In 2018, the prefecture-level city of Laiwu was abolished, which assigned to Jinan. Therefore, Laiwu was still studied as a prefecture-level city for the study period was from 2005 to 2014 . Shandong Province covers an area of 155,800 square kilometers and has a permanent resident population of 100.7 million.

\section{Data Source}

Notifiable respiratory infectious diseases data for this study were derived from the China Information System for Disease Control and Prevention. All respiratory infectious diseases cases were defined base on the diagnostic criteria and principles of management for notifiable infection diseases issued by National Health Commission of the People's Republic of China. Only the cases confirmed by both clinically and laboratory tests, including microscopic examination and biochemical identification, were included in the study. Demographic data of Shandong Province were collected from the Sixth National Population Census in 2010 and the Center for Public Health Science Data in China (http://www.phsciencedata.cn/).

\section{Study design and statistical analysis}

Spatial, temporal and spatiotemporal analysis were performed to understand the temporal and spatial distribution characteristics of respiratory infectious diseases and determine its spatial-temporal clustering in Shandong province. We examined the temporal and spatial distribution characteristics of respiratory infectious diseases in the following three-step process.

Firstly, Time series was applied to describe the temporal distribution characteristics of respiratory infectious diseases. Monthly incidence rate of each respiratory infectious disease during the study period (2005-2014) was exhibited to observe the temporal trends of each respiratory infectious disease. We used the ArcGIS 10.4 software to produce the thematic maps of the average annual incidence rate of each respiratory infectious disease. The average annual incidence rate was graded by Natural Break (Jenks), which was divided into 5 levels. The Natural Break (Jenks) can identify break points by picking the class breaks that best group similar values and maximize the differences between classes[19]. Since the onset of respiratory infectious diseases is a low-probability event with aggregation and instability in China, empirical Bayes can make statistical adjustment according to the principle that large population is more stable than small population, so as to obtain more stable clustering and make the results more accurate and reliable[20]. In our study, spatial empirical Bayesian smoothing was used to adjust the average annual incidence rate of each respiratory infectious disease, and the estimation rate based on spatial weight matrix was closer to the real situation of geographical distribution of diseases[20]. GeoDa 
1.10 software was used to apply spatial empirical Bayesian smoothing and map the excess risk (ER) of each respiratory infectious disease.

Secondly, spatial autocorrelation analysis was used to identify the spatial clustering of each respiratory infectious disease in Shandong Province. Moran's / is an important indicator to analyze the spatial distribution characteristics of disease cases. Global Moran's / was used to detect whether the significant spatial autocorrelation regions of each respiratory infectious disease in Shandong Province existed[21]. Then local Moran's / was further used to clarify the patterns of spatial autocorrelation in spatial units. The Moran's / rangs from -1 to 1 , which indicates a positive correlation when the value is $>0$ and a negative correlation when the value is $<0$ at a statistically significant level. By using local indicators of spatial association (LISA) map[22, 23], four different spatial clustering modes were demonstrated, which were: High-High ( $\mathrm{HH}$, high-incidence regions surrounded by high-incidence regions, i.e., hotspots), Low-Low (LL, low-incidence regions surrounded by low-incidence regions, i.e., coldspots), High-Low (HL, high-incidence regions surrounded by low-incidence regions) and Low-High ( $\mathrm{LH}$, low-incidence regions surrounded by high-incidence regions). Spatial autocorrelation analysis was performed using the univariate (local) Moran's / tool in the GeoDa 1.10 software. The significance level was set at $P<0.05$ and $95 \%$ confidence level $(\mathrm{Cl})$ with the number of simulations at 999 .

Lastly, the space-time scan statistic was adopted to explore the spatial-temporal clusters of each respiratory infectious disease. In this study, the monthly incidence was taken as the clustering unit and the county as the minimum spatial unit. We used a cylindrical scanning window for probe scanning. The maximum spatial cluster size and maximum temporal cluster size were all set to $50 \%$. For each window, the expected number of cases can be inferred by using the discrete Poisson model with the observed number of cases and the number of the population within/outside the moved windows (the potential clusters) of candidate regions during candidate time[24, 25]. The relative risk (RR) was calculated by the ratio of the observed number to the expected number within the windows and outside the windows. Loglikelihood ratio (LLR) was employed to identify the special clusters by comparing the observed incidence with expected one, and the $P$ value of LLR was obtained by the Monte Carlo method with the simulation time 999[24]. The window with the largest LLR value was defined as the most likely cluster, and other windows that contained clusters with statistically significant LLR values were defined as the first secondary cluster, the second secondary cluster, etc. The analysis process was carried out using the software SaTScan 9.4.4 and the scanning results were visualized by ArcGIS 10.4 software.

\section{Results}

\section{Descriptive analysis for respiratory infectious diseases}

There were 537,506 cases of respiratory infectious diseases in total in Shandong Province over the study period. Table 1 shows the incidence of each respiratory infectious disease in study area during the study period. No cases were notified in diphtheria during the study period. Ten other respiratory infectious disease types were reported during the study period, with annual average incidences ranging from 0.001 
to 36.450 per 100,000 . It was difficult to analyze the spatial-temporal clusters of diphtheria and severe acute respiratory syndrome (SARS). Therefore, the two diseases were not incorporated in the next analysis.

\section{The temporal distribution of respiratory infectious diseases}

The monthly incidence of each respiratory infectious disease is shown in Fig. 1. The time series of influenza A (H1N1) indicated outbreaks of influenza A (H1N1) occurred in the winter of 2009, with the highest monthly incidence (1.96 per 100,000) in November 2009 and sporadic in other years (See Fig. 1A). The monthly incidence of measles had an obvious seasonal increase in spring and peaked in March 2008 (2.13 per 100,000). The incidence of measles dropped significantly after 2010 and then stabilized (See Fig. 1B). The monthly incidence rate of tuberculosis is between 4 per 100,000 and 5 per 100,000 , which peaked in late winter and early spring, mainly from January to April. The incidence of tuberculosis gradually decreased since 2014 (See Fig. 1C). From 2005 to 2014, the monthly incidence of meningococcal meningitis showed a significant downward trend, with peaking in late spring, early summer (May-June) and winter (December, See Fig. 1D). The incidence of pertussis was stable and low during the study period ( $<0.1$ per 100,1000, See Fig. 1E). The monthly incidence of scarlet fever remained relatively stable in 2005-2009, but began to increase rapidly since 2010. The incidence of scarlet fever showed a bimodal seasonal pattern with a peak occurring in summer and a peak occurring in winter (See Fig. 1F). The incidence of influenza rapidly increased since 2009 with strong seasonality in winter, and its peak occurred in December 2009 (1.39 per 100,000, See Fig. 1G). The incidence of mumps was relatively stable during the study period with a fluctuation in 2012 (See Fig. 1H). The time series indicated outbreaks of rubella occurred in March 2006 with the monthly incidence rate of 1.65 per 100,000 (See Fig. 11).

\section{The spatial distribution of respiratory infectious diseases}

The spatial distribution of respiratory infectious diseases in Shandong Province is shown in Fig. 2 and Fig. S2 (See Supplementary Fig. 2, Additional File 1). The incidence of influenza A (H1N1) was relatively dispersed, and the top three counties with the higher incidence were Sifang District in Qingdao (10.6 per 100,000), Fushan District in Yantai (8.6 per 100,000) and Taishan District in Taian (6.1 per 100,000, See Fig. 2A). After spatial empirical Bayesian smoothing, the highly incidence of influenza A (H1N1) occurred in Jinan and Northwest Shandong (See Supplementary Fig. 2A, Additional File 1). There were 13 counties in the incidence of influenza A (H1N1) with ER above 4, such as Wudi County and Huimin County in Dezhou, and there were 26 counties with ER greater than 1 and less than 4 (See Supplementary Fig. 3A, Additional File 1). The incidence of measles is high in Northwest and South Shandong with average annual incidence rate of 6.5 per 100,1000 to 15.0 per 100,1000 (See Fig. 2B). The result of measles after Bayesian smoothing was consistent with that of Natural Break classification (See Supplementary Fig. 2B, Additional File 1). There were 60 counties in the incidence of measles with ER greater than 1 (See Supplementary Fig. 3B, Additional File 1). We can see that tuberculosis struck all counties, with a higher incidence in West Shandong, Northwest Shandong and Southeast Shandong from the thematic map and 
Bayesian smoothing map of tuberculosis (See Fig. 2C \& See Supplementary Fig. 2C, Additional File 1). The highest annual incidence of tuberculosis was in Dongping County in Taian (62.7 per 100,000), followed by Qingyun County in Dezhou (60.6 per 100,000). ER above 1 covered 72 counties in the ER map of tuberculosis (See Supplementary Fig. 3C, Additional File 1). The incidence of meningococcal meningitis was relatively low in the whole Shandong, with a higher incidence in Liaocheng, Heze, Jining and Linyi (See Fig. 2D). After Bayesian smoothing, the incidence of meningococcal meningitis decreased in Northern and Central Shandong (See Supplementary Fig. 2D, Additional File 1). The ER map of meningococcal meningitis showed 55 counties with ER greater than 1 (See Supplementary Fig. 3D, Additional File 1). The incidence of pertussis was also relatively low in Shandong province, with a higher incidence in Northwestern Shandong and Southern Shandong (See Fig. 2E). After Bayesian smoothing, it showed that the incidence of pertussis decreased in Pingyuan County in Dezhou and increased in Central Shandong (See Supplementary Fig. 2E, Additional File 1). The ER of 49 counties was greater than 1 (See Supplementary Fig. 3E, Additional File 1). The spatial distribution of scarlet fever showed that the high incidence of scarlet fever was distributed in the eastern coastal area and Central Shandong, with a high incidence in Jinan, Zibo, Yantai and Yantai (See Fig. 2F). The result of scarlet fever after Bayesian smoothing was consistent with that of Natural Break classification (See Supplementary Fig. 2F, Additional File 1). The ER of 45 counties was greater than 1 (See Supplementary Fig. 3F, Additional File 1). The spatial distribution of influenza showed that West Shandong (such as Liaocheng, Dezhou, Heze and Jinan) had higher incidence of influenza than others and the counties with the top incidences of influenza were Pingyuan County in Dezhou (33.3 per 100,000), Central District in Jining (31.7 per 100,000) and Yucheng County-level city in Dezhou (29.2 per 100,000, Fig. 2 G \& See Supplementary Fig. 2G, Additional File 1). There were 45 counties in the incidence of influenza with ER above 1 (See Supplementary Fig. 3G, Additional File 1). A higher incidence of mumps was in Binzhou, Jinan and Taian. The top average annual incidences of mumps were in Guorao County in Dongying (44.3 per 100,000), Shizhong District in Jining (41.6 per 100,000) and Rencheng County in Jining (35.0 per 100,000, Fig. 2H \& See Supplementary Fig. 2H, Additional File 1). The ER map of mumps showed 59 counties with ER greater than 1 (See Supplementary Fig. 3H, Additional File 1). The spatial distribution of rubella showed that the areas with high incidence of rubella were in northern Shandong and Middle Shandong, such as Binzhou, Dongying, Jinan and Binzhou. The highest average annual incidence of rubella was in Huantai County in Zibo (13.6 per 100,000, Fig. 2I \& See Supplementary Fig. 2l, Additional File 1). The ER map of rubella showed there were 69 counties with ER above 1 . Among them, the ERs of rubella in Changle County, Gaoqing County and Changdao County were higher than other counties (See Supplementary Fig. 3l, Additional File 1).

\section{Spatial autocorrelation analysis}

The result of global spatial autocorrelation analysis showed that the Moran's / of average annual incidence of each respiratory infectious disease in Shandong Province ranged from 0.062 to 0.353 . This result indicated that there was an evident spatial correlation blinding the cases of respiratory infectious diseases except meningococcal meningitis (Table 2). Only those counties whose local Moran's / had reached the significance level of 0.05 were presented on the LISA cluster maps. The local spatial 
autocorrelation analysis showed that the incidence of each respiratory infectious disease in Shandong Province had an obvious clustering (See Fig. 3). The LISA result of influenza A (H1N1) showed that there were $5 \mathrm{HH}$ cluster regions (i.e., hotspots), which were mainly concentrated in Qingdao and Jinan. While there were $14 \mathrm{LL}$ cluster regions (i.e., coldspots), which were mainly concentrated in Yantai, Weifang and Linyi (See Fig. 3A). As seen in Fig. 3A, Gaomi, Jimo, Longkou and Penglai were LH cluster regions, and municipal district of Heze, municipal district of Jining, municipal district of Dezhou were HL cluster regions. For measles, the $23 \mathrm{HH}$ cluster regions were mainly located in South Shandong and Dezhou, while the $34 \mathrm{LL}$ cluster regions were mainly located in Northeast Shandong (See Fig. 3B). As seen in Fig. 3B, Zaozhuang and Linyi displayed a LH cluster feature, and municipal district of Zibo, Jimo, municipal district of Yantai displayed a HL cluster feature. We identified $24 \mathrm{HH}$ cluster regions for tuberculosis, which were primarily concentrated in Binzhou, Liaocheng and Linyi. While 30 LL cluster regions were identified for tuberculosis, which were primarily concentrated in Weifang, Zibo and Yantai (See Fig. 3C). As seen in Fig. 3C, the LH cluster regions of tuberculosis were scattered in Dezhou, Heze and Jining, and the $\mathrm{HL}$ cluster regions were mainly distributed in Yantai. For meningococcal meningitis, we identified $4 \mathrm{HH}$ cluster regions, $18 \mathrm{LL}$ cluster regions, $8 \mathrm{LH}$ cluster regions and $6 \mathrm{HL}$ cluster regions (See Fig. 3D). There were $20 \mathrm{HH}$ cluster regions for pertussis, mainly distributed in North Liaocheng and South Dezhou, Ju County, Juancheng County, Yuncheng County, Cangshan County and Central District of Zaozhuang. While, there were $46 \mathrm{LL}$ cluster regions, mainly distributed in Northeast Shandong. We also identified $7 \mathrm{LH}$ cluster regions and a HL cluster region (See Fig. 3E). The $8 \mathrm{HH}$ cluster regions and $31 \mathrm{LL}$ cluster regions for scarlet fever were mostly from counties in Qingdao, Weifang, Laiwu, South Shandong and Dezhou. Some counties in Qingdao, Weifang, Jinan, Rizhao and Dezhou were identified for LH or HL cluster regions (See Fig. 3F). The LISA result of influenza showed that there were $14 \mathrm{HH}$ cluster regions, which were mainly concentrated in Dezhou and Jining. While there were 25 LL cluster regions, which were mainly concentrated in Yantai, Weifang and Qingdao (See Fig. 3G). And $10 \mathrm{LH}$ cluster regions and $3 \mathrm{HL}$ cluster regions were identified for influenza (See Fig. 3G). For mumps, the $10 \mathrm{HH}$ cluster regions were mainly located in Dongying, while the $22 \mathrm{LL}$ cluster regions were mainly located in Yantai, Weifang, Rizhao (See Fig. 3H). For rubella, we identified 7 HH cluster regions, 21 LL cluster regions, 8 LH cluster regions and $5 \mathrm{HL}$ cluster regions (See Fig. 3I).

\section{Spatial-temporal scanning analysis}

Fig. 4 and Table S1(See Supplementary Table 1, Additional File 1) show that respiratory infectious diseases in Shandong Province had a non-random spatial and temporal distribution. The most likely cluster times for respiratory infectious diseases were in winter-and-spring during the study period except pertussis and mumps (See Supplementary Table 1, Additional File 1). But the most likely clusters in space were somewhat different for different respiratory infectious diseases (See Fig. 4).

The most likely cluster time for influenza A (H1N1) was November 2009, and the most likely cluster areas were mainly concentrated in Central Shandong and Eastern Shandong (74 counties in total), such as Zhucheng County-level city, Penglai County-level city, Rongcheng County-level city, Lijin County, municipal district of Weihai, etc. The cluster center was located at $36^{\circ} 77^{\prime} \mathrm{N}, 119^{\circ} 22^{\prime} \mathrm{E}$, the cluster radius was 199.83 
$\mathrm{km}$, the average annual rate within this range was 27.1 per 100,000 , and the RR was $39.79(P<0.001$, See Fig. 4A).

The most likely cluster time for measles was March 2008, and the most likely cluster areas were mainly concentrated in Southwest Shandong (66 counties in total), such as Laoling County-level city, Ningjin County, Huimin County, Ling County, Shanghe County, etc. The cluster center was located at $35^{\circ} 28^{\prime} \mathrm{N}$, $115^{\circ} 08^{\prime} \mathrm{E}$, the cluster radius was $292.83 \mathrm{~km}$, the average annual rate within this range was $34.0 \mathrm{per}$ 100,000 , and the RR was $13.38(P<0.001$, See Fig. 4B).

The most likely cluster time for tuberculosis was January 2007, and the most likely cluster areas were mainly concentrated in Central Shandong and Northwest Shandong (58 counties in total), such as Wucheng County, Xiajin County, Pingyuan County, Gaotang County, Ling County, etc. The cluster center was located at $37^{\circ} 17^{\prime} \mathrm{N}, 116^{\circ} 43^{\prime} \mathrm{E}$, the cluster radius was $183.32 \mathrm{~km}$, the average annual rate within this range was 82.1 per 100,000 , and the RR was 2.32 ( $P<0.001$, See Fig. 4 C).

The most likely cluster time for meningococcal meningitis was February 2005, and the most likely cluster areas were mainly concentrated in Southwest Shandong (56 counties in total), such as municipal district of Dezhou, Qihe County, municipal district of Jinan, Linqing County-level city, Changqing County, etc. The cluster center was located at $34^{\circ} 80^{\prime} \mathrm{N}, 116^{\circ} 08^{\prime} \mathrm{E}$, the cluster radius was $230.36 \mathrm{~km}$, the average annual rate within this range was 0.5 per 100,000 , and the RR was 23.49 ( $P<0.001$, See Fig. 4D). We also obtained one secondary cluster for meningococcal meningitis, which consisted of 37 cluster counties. The secondary spatial-temporal clusters were mainly in Central Shandong and Eastern Shandong (such as Lijin County, Laixi County-level city, Boxing County, Huantai County, municipal district of Zibo, etc.) during March 2005 (See Fig. 4D \& See Supplementary Table 1, Additional File 1).

The most likely cluster time for pertussis was July 2007, and the most likely cluster areas were mainly concentrated in Southwest Shandong (22 counties in total), such as Yutai County, Jinxiang County, Tengzhou County-level city, etc. The cluster center was located at $35^{\circ} 00^{\prime} \mathrm{N}, 116^{\circ} 65^{\prime} \mathrm{E}$, the cluster radius was $98.58 \mathrm{~km}$, the average annual rate within this range was 3.5 per 100,000, and the RR was 21.44 $(P<0.001$, Fig. 4E). We also obtained two secondary clusters for pertussis, which consisted of 12 cluster counties. The secondary spatial-temporal clusters were mainly in Northwest Shandong (such as Pingyuan County, Ling County, Yucheng County-level city, etc.) during June 2010 and June 2005 (See Fig. 4E \& See Supplementary Table 1, Additional File 1).

The most likely cluster time for scarlet fever was December 2011, and the most likely cluster areas were mainly concentrated in Central Shandong and North Shandong (64 counties in total), such as Zibo, Binzhou, Laiwu, Jinan, etc. The cluster center was located at $36^{\circ} 70^{\prime} \mathrm{N}, 118^{\circ} 82^{\prime} \mathrm{E}$, the cluster radius was $168.54 \mathrm{~km}$, the average annual rate within this range was 21.2 per 100,000 , and the RR was 9.34 $(P<0.001$, See Fig. 4F).

The most likely cluster time for influenza were November 2009, and the most likely cluster areas were concentrated in Shizhong District and Rencheng District in Jining. The cluster center was located at 
$35^{\circ} 42^{\prime} \mathrm{N}, 116^{\circ} 58^{\prime} \mathrm{E}$, the cluster radius was $0 \mathrm{~km}$, the average annual rate within this range was 751.6 per 100,000 , and the RR was 310.29 ( $P<0.001$, See Fig. 4G). We also obtained three secondary clusters for influenza, which consisted of 77 cluster counties. The secondary spatial-temporal clusters were mainly in Northwest Shandong, Penglai County-level city, municipal district of Qingdao, Jiaozhou County-level city, etc. during December 2013, March 2010 and September 2009 (See Fig. 4G \& See Supplementary Table 1, Additional File 1).

The most likely cluster time for mumps was June 2012, and the most likely cluster areas were mainly concentrated in Central Shandong and Northwest Shandong (72 counties in total), such as Lijin County, Qingyun County, Laoling County-level city, Ningjin County, Yangxin County, etc. The cluster center was located at $37^{\circ} 73^{\prime} \mathrm{N}, 117^{\circ} 23^{\prime} \mathrm{E}$, the cluster radius was $239.96 \mathrm{~km}$, the average annual rate within this range was 51.8 per 100,000, and the RR was $4.84(P<0.001$, See Fig. $4 \mathrm{H})$. We also obtained one secondary cluster for mumps, which consisted of 1 cluster county. And the cluster time was July 2005 (See Fig. 4H \& See Supplementary Table 1, Additional File 1).

The most likely cluster time for rubella was May 2005, and the most likely cluster areas were mainly concentrated in West Shandong (56 counties in total), such as Xiajin County, Gaotang County, Linqing County-level city, Wucheng County, Pingyuan County, etc. The cluster center was located at $36^{\circ} 85^{\prime} \mathrm{N}$, $115^{\circ} 70^{\prime} \mathrm{E}$, the cluster radius was $230.36 \mathrm{~km}$, the average annual rate within this range was $190.5 \mathrm{per}$ 100,000 , and the RR was 122.07 ( $P<0.001$, See Fig. 4I).

\section{Discussion}

This study has, for the first time, revealed the spatial-temporal epidemiology of respiratory infectious diseases in Shandong Province extensively and systematically using monitoring data. The scan statistical technique can provide means to detect spatiotemporal distribution of respiratory infectious diseases, as well as to identify high-risk areas. Spatial-temporal analysis of respiratory infectious diseases in our study is not only helpful to understand the epidemic characteristics of respiratory infectious diseases in Shandong province, but also provides scientific basis for health policy makers, public health professionals and clinicians to control and prevent respiratory infectious diseases.

The results of the time series of respiratory infectious diseases indicated that the incidence of respiratory infectious diseases in Shandong Province has obvious seasonality, with peaking in winter and spring and a few respiratory infectious diseases in early summer, which may be related to climatic factors[26-29]. In addition, we found that the incidence of measles, tuberculosis and meningococcal meningitis showed a downward trend, and the incidence of influenza $A(\mathrm{H} 1 \mathrm{~N} 1)$ and rubella showed sporadic and have an outbreak trend occasionally. However, the incidence of scarlet fever, mumps and influenza had been rising in recent years in Shandong Province, which suggested to improve the awareness of prevention and treatment of respiratory infectious diseases. The temporal distribution characteristics of respiratory infectious diseases were consistent with the findings of Mao et al[18]. The reasons for the increased incidence of these respiratory infections may be as follows: some respiratory infectious pathogens 
mutate, which can increase susceptibility of humans, such as the emergence of new influenza virus[30]; and some respiratory infections are asymptomatic or subclinical infections, which have the potential to cause outbreaks.

The spatial distribution characteristics of different respiratory infectious diseases are different. Our study showed that the incidence of respiratory infectious diseases in Shandong Province is mainly in West Shandong and Central Shandong, followed by South Shandong. The incidence of respiratory infectious diseases in coastal areas is relatively well controlled, indicating that the distribution characteristics of respiratory infectious diseases are closely related to geographical and environmental factors[31, 32]. The analysis of this study showed that there is obvious spatial clustering of each respiratory infectious disease. The hotspots areas of influenza A (H1N1) were mainly in Qingdao and Jinan, the hotspots areas of measles were mainly in South Shandong and Dezhou, the hotspots areas of tuberculosis were mainly in Binzhou, Liaocheng and Linyi, the hotspots areas of meningococcal meningitis were mainly in Heze and Linyi, the hotspots areas of pertussis are mainly in North Liaocheng, South Dezhou, Ju County, Juancheng County, Yuncheng County, Cangshan County and Central District of Zaozhuang, the hotspots areas of scarlet fever were mainly in Qingdao, Laiwu and Qingzhou County-level city, the hotspots areas of influenza were mainly in Dezhou and Jining, the hotspots areas of mumps was mainly in Dongying, and the hotspots areas of rubella was mainly in Bingzhou. The high-risk areas have been identified for some respiratory infectious diseases in Shandong Province in several studies, for example, measles[33, 34], tuberculosis[35], pertussis[36], and mumps[37]. And the results of these studies are similar to our findings. The corresponding effective control strategies, such as improving vaccine coverage of susceptible population, enhance health education and environmental management, are important for respiratory infectious diseases control and should therefore be implemented by the administrative departments of public health.

The temporal-spatial clustering analysis identified the most likely cluster and several secondary clusters for each respiratory infectious disease, indicating that the incidence of respiratory infectious diseases is increasing. And the time windows were mainly concentrated in winter-and-spring. The most likely cluster and several secondary clusters for respiratory infectious diseases differ with the hotspot analysis, because the hotspot analysis did not consider the time factor and arbitrarily conducted static scale selection. The spatial-temporal scanning method achieved effective temporal and spatial integration, which was able to evaluate the clustering in different time windows to achieve a dynamic, threedimensional and multi-scale analysis[38]. For influenza A (H1N1), especially in Central Shandong and Eastern Shandong, local public-health authority should simultaneously strengthen influenza A (H1N1) surveillance and prevention among high-risk groups. For measles, especially in the 66 counties of Southwest Shandon, local public-health authority should simultaneously strengthen measles surveillance and the coverage rate of measles vaccine in floating population. For tuberculosis, especially in Central Shandong and Northwest Shandong, local public-health authority should simultaneously strengthen health promotion for tuberculosis and surveillance in the high-risk population. For meningococcal meningitis, especially in the 56 counties of Southwest Shandong and 37 counties of Central Shandong and Eastern Shandong, local public-health authority should simultaneously strengthen meningococcal 
meningitis surveillance and improve the coverage of meningococcal meningitis vaccine in densely populated areas. For pertussis, especially in 22 counties of Southwest Shandong and 12 counties of Northwest Shandong, local public-health authority should simultaneously strengthen pertussis surveillance and the coverage of diphtheria-pertussis-tetanus vaccine among high-risk groups. For scarlet fever, especially in Central Shandong and North Shandong, the health department should strengthen the monitoring and prevention of scarlet fever in high-risk groups and peak periods of scarlet fever. For influenza, especially in Northwest Shandong and Shizhong District and Rencheng District in Jining, the health department should strengthen the monitoring and health publicity and education among influenza season. For mumps, especially in Central Shandong and Northwest Shandong, the health department should simultaneously strengthen mumps surveillance and prevention among high-risk population. For rubella, especially in 56 counties of West Shandong, the health department should simultaneously strengthen rubella surveillance and health promotion among high-risk population.

Several limitations of this study should be acknowledged. First, the spatial-temporal scanning was very useful to explore spatially aggregated data and to highlight the riskiest areas to conduct more accurate analysis[39]. But we must note that ecologic bias was inevitable in any ecological study[40]. For example, we took counties as the analysis space unit in this study to conduct the spatial-temporal cluster of respiratory infectious diseases. The results could only reflect the aggregation of the county, the urban and rural distribution characteristics of respiratory infectious diseases in Shandong Province could not be inferred. Second, local Moran's / could reflect the clustering of the respiratory infectious disease in spatial pattern, but it might be disturbed by population fluctuation. And some irregular counties could limit the power of circular scan statistics. Third, because Chinese Center for Disease Control and Prevention only shared with us the data from 2005-2014, we failed to analyze the temporal and spatial distributions of respiratory infectious diseases from 2015 to 2019, which might be slightly different from 2014 to 2019 . Finally, this study did not evaluate potential environmental risk factors and population characteristics associated with the different temporal and spatial distributions of respiratory infectious diseases. Therefore, it is important to conduct further studies to identify the geographical risk factors and determine precise local prevention and control measures.

\section{Conclusions}

In summary, our study analyzed the epidemiological characteristics of respiratory infectious diseases in Shandong Province and revealed that different respiratory infectious diseases display different trends. Our study confirmed the spatial autocorrelation of respiratory infectious diseases, and spatial-temporal clusters with high risk of respiratory infectious diseases in Shandong Province. Different respiratory infectious diseases had different hotspots and temporal and spatial clusters. In general, measles, tuberculosis, meningococcal meningitis, pertussis, influenza, mumps and rubella were mainly concentrated in Northwest and Southwest Shandong, and influenza A (H1N1) and scarlet fever were mainly distributed in Northern Shandong. The cluster time of respiratory infectious diseases was in winter, spring and early summer. 


\section{Abbreviations}

DALYs: Disability adjusted life years; GBD: the Global Burden of Disease; RSV: Respiratory syncytial virus; GIS: Geographic information systems; ER: Excess risk; LISA: Local indicators of spatial association; HH: High-incidence regions surrounded by high-incidence regions; LL: Low-incidence regions surrounded by low-incidence regions; HL: High-incidence regions surrounded by low-incidence regions; LH: Lowincidence regions surrounded by high-incidence regions; Cl: Confidence level; RR: Relative risk; LLR: Loglikelihood ratio; SARS: Severe acute respiratory syndrome

\section{Declarations}

\section{Ethics approval and consent to participate}

Not applicable.

\section{Consent for publication}

Not applicable.

\section{Availability of data and materials}

The datasets used during the current study are available from the corresponding author on reasonable request.

\section{Competing interests}

The authors declare that they have no competing interests.

\section{Funding}

This study was supported by the Academic Promotion Program of Shandong First Medical University (2019RC010), the Shandong Province Higher Educational Young and Innovation Technology Supporting Program (2019KJL004) and the PhD Scientific Research Staring Foundation of Shandong First Medical University (Xuena Liu).

\section{Author's contributions}

GD and XL (Xuena Liu) designed the study. XL (Xuewen Li), SZ and MC collated the data. XL (Xiaomei Li) and $X X$ analysed the data. GD, DC, XL (Xiaomei Li) and YZ drafted the manuscript. All authors had input on the manuscript draft. GD and XL (Xuena Liu) provided valuable edits. All authors interpreted the results, revised the report and approved the final version.

\section{Acknowledgments}


We thank Chinese Center for Disease Control and Prevention and Data center for Institute of Geographic Sciences and Natural Resources Research of China sharing with us the data needed for this study.

\section{References}

1. Kassebaum NJ, Smith AGC, Bernabé E, Fleming TD, Reynolds AE, Vos T, et al. Global, Regional, and National Prevalence, Incidence, and Disability-Adjusted Life Years for Oral Conditions for 195 Countries, 1990-2015: A Systematic Analysis for the Global Burden of Diseases, Injuries, and Risk Factors. J Dent Res. 2017;96(4):380-7.

2. Bureau of Disease Control and Prevention, 2020. Profiling of the Epidemic Situation of Notifiable Infectious Diseases in China in 2019. http://www.nhc.gov.cn/jkj/s3578/202004/b1519e1bc1a944fc8ec176db600f68d1.shtml (Accessed 11 Jul 2020) (in Chinese).

3. Mukherjee S. Emerging Infectious Diseases: Epidemiological Perspective. Indian J Dermatol. 2017;62(5):459-67.

4. World Health Organization, 2020. Coronavirus disease 2019 (COVID-19) Situation Report. https://www.who.int/emergencies/diseases/novel-coronavirus-2019/situation-reports (Accessed 18 Jun 2020).

5. Yang Z, Wang N, Ding QY. Distribution difference of respiratory infectious disease epidemic in China. Foreign Medical Science Section of Medgeography. 2018:39(01):1-5.

6. Angulo J, Yu HL, Langousis A, Kolovos A, Wang J, Madrid AE, et al. Spatiotemporal infectious disease modeling: a BME-SIR approach. PLoS One. 2013;8(9):e72168.

7. Zhang Y, Shen Z, Ma C, Jiang C, Feng C, Shankar N, et al. Cluster of human infections with avian influenza A (H7N9) cases: a temporal and spatial analysis. Int J Environ Res Public Health. 2015;12(1):816-28.

8. Li Z, Fu J, Lin G, Jiang D. Spatiotemporal Variation and Hotspot Detection of the Avian Influenza A(H7N9) Virus in China, 2013(-)2017. Int J Environ Res Public Health. 2019;16(4):648.

9. Dong W, Yang K, Xu QL, Yang YL. A Predictive Risk Model for A(H7N9) Human Infections Based on Spatial-Temporal Autocorrelation and Risk Factors: China, 2013-2014. Int J Environ Res Public Health. 2015;12(12):15204-21.

10. Chowell G, Viboud C, Munayco CV, Gomez J, Simonsen L, Miller MA, et al. Spatial and temporal characteristics of the 2009 A/H1N1 influenza pandemic in Peru. PLoS One. 2011;6(6):e21287.

11. Li L, Xi Y, Ren F. Spatio-Temporal Distribution Characteristics and Trajectory Similarity Analysis of Tuberculosis in Beijing, China. Int J Environ Res Public Health. 2016;13(3):291.

12. Huang L, Li XX, Abe EM, Xu L, Ruan Y, Cao CL, et al. Spatial-temporal analysis of pulmonary tuberculosis in the northeast of the Yunnan province, People's Republic of China. Infect Dis Poverty. 2017;6(1):53. 
13. Chen J, Qiu Y, Yang R, Li L, Hou J, Lu K, et al. The characteristics of spatial-temporal distribution and cluster of tuberculosis in Yunnan Province, China, 2005-2018. BMC Public Health. 2019;19(1):1715.

14. Rao HX, Shi $X Y$, Zhang $X$. Using the Kulldorff's scan statistical analysis to detect spatio-temporal clusters of tuberculosis in Qinghai Province, China, 2009-2016. BMC Infect Dis. 2017;17(1):578.

15. Liu MY, Li QH, Zhang YJ, Ma Y, Liu Y, Feng W, et al. Spatial and temporal clustering analysis of tuberculosis in the mainland of China at the prefecture level, 2005-2015. Infect Dis Poverty. 2018;7(1):106.

16. Li WT, Feng RH, Li T, Du YB, Zhou N, Hong XQ, et al. Spatial-temporal analysis and visualization of scarlet fever in mainland China from 2004 to 2017. Geospat Health. 2020;15(1):10.4081/gh.2020.831.

17. Yu G, Yang R, Wei Y, Yu D, Zhai W, Cai J, et al. Spatial, temporal, and spatiotemporal analysis of mumps in Guangxi Province, China, 2005-2016. BMC Infect Dis. 2018;18(1):360.

18. Mao Y, He R, Zhu B, Liu J, Zhang N. Notifiable Respiratory Infectious Diseases in China: A SpatialTemporal Epidemiology Analysis. Int J Environ Res Public Health. 2020;17(7):2301.

19. Xiaofeng L, Yi Q, Diqiang L, Shirong L, Xiulei W, Bo W, et al. Habitat evaluation of wild Amur tiger (Panthera tigris altaica) and conservation priority setting in north-eastern China. J Environ Manage. 2011;92(1):31-42.

20. Owusu-Edusei K, Jr., Owens CJ. Monitoring county-level chlamydia incidence in Texas, 2004 - 2005: application of empirical Bayesian smoothing and Exploratory Spatial Data Analysis (ESDA) methods. Int J Health Geogr. 2009;8:12.

21. Moran PAP. Notes on continuous stochastic phenomena. Biometrika. 1950:37(1-2):17-23.

22. Amelin L. Local indicators of spatial association-LISA. Geogr Anal. 1995:27(2):93-115.

23. Ord JK, Getis A. Local spatial autocorrelation statistics: distributional issues and an application. Geogr Anal. 1995: 27(4):286-306.

24. Kulldorff M, Athas WF, Fever EJ, Miller BA, Key CR. Evaluating cluster alarms: a space-time scan statistic and brain cancer in Los Alamos, New Mexico. Am J Public Health. 1998:88(9):1377-80.

25. Kulldorff M. Prospective time periodic geographical disease surveillance using a scan statistic. J R Stat Soc Ser A Stat Soc. 2001:164(1):61-72.

26. Zha WT, Li WT, Zhou N, Zhu JJ, Feng R, Li T, et al. Effects of meteorological factors on the incidence of mumps and models for prediction, China. BMC Infect Dis. 2020;20(1):468.

27. Khaliq A, Batool SA, Chaudhry MN. Seasonality and trend analysis of tuberculosis in Lahore, Pakistan from 2006 to 2013. J Epidemiol Glob Health. 2015;5(4):397-403.

28. Li Y, Ye X, Zhou J, Zhai F, Chen J. The association between the seasonality of pediatric pandemic influenza virus outbreak and ambient meteorological factors in Shanghai. Environ Health. 2020;19(1):71.

29. Peng L, Zhao X, Tao Y, Mi S, Huang J, Zhang Q. The effects of air pollution and meteorological factors on measles cases in Lanzhou, China. Environ Sci Pollut Res Int. 2020;27(12):13524-33. 
30. Wille M, Holmes EC. The Ecology and Evolution of Influenza Viruses. Cold Spring Harb Perspect Med. 2020;10(7):a038489.

31. Carrasco-Escobar G, Schwalb A, Tello-Lizarraga K, Vega-Guerovich P, Ugarte-Gil C. Spatio-temporal co-occurrence of hotspots of tuberculosis, poverty and air pollution in Lima, Peru. Infect Dis Poverty. 2020;9(1):32.

32. Yu G, Yang R, Yu D, Cai J, Tang J, Zhai W, et al. Impact of meteorological factors on mumps and potential effect modifiers: An analysis of 10 cities in Guangxi, Southern China. Environ Res. 2018;166:577-87.

33. Zhu YH, Xu Q, Lin HL, Yue DH, Song LZ, Wang CY, et al. Spatiotemporal Analysis of Infant Measles Using Population Attributable Risk in Shandong Province, 1999-2008. PLoS One. 2013;8(11):e79334.

34. Li X, Kang D, Zhang Y, Wei G, Liu W, Fang L, et al. Epidemic trend of measles in Shandong Province, China, 1963-2005. Public Health. 2012;126(12):1017-23.

35. Li YW, Cheng J, Wang H, Zhao F, Li XX, Tao WW, et al. [Spatial-temporal analysis of pulmonary tuberculosis in Shandong province, 2015]. Zhonghua Liu Xing Bing Xue Za Zhi. 2016;37(9):1257-61.

36. Zhang Y, Bambrick H, Mengersen $K$, Tong S, Feng L, Zhang L, et al. Resurgence of Pertussis Infections in Shandong, China: Space-Time Cluster and Trend Analysis. Am J Trop Med Hyg. 2019;100(6):1342-54.

37. Li R, Cheng S, Luo C, Rutherford S, Cao J, Xu Q, et al. Epidemiological Characteristics and SpatialTemporal Clusters of Mumps in Shandong Province, China, 2005-2014. Sci Rep. 2017;7:46328.

38. Wang PA, Luo WH, Bai YP. Comparative analysis of aggregation detection based on spatial autocorrelation and spatial temporal scan statistics. Human Geography. 2012:27:119-27.

39. Liu YX, Wang XJ, Pang CK, Yuan ZS, Li HK, Xue FZ. Spatio-temporal analysis of the relationship between climate and hand, foot, and mouth disease in Shandong province, China, 2008-2012. BMC Infect Dis. 2015;15:146.

40. Morgenstern H. Ecologic studies in epidemiology: concepts, principles, and methods. Annu Rev Public Health. 1995;16:61-81.

\section{Tables}


Table 1

The incidence of each respiratory infectious disease in Shandong Province during the study period.

\begin{tabular}{|c|c|c|c|}
\hline Disease & Time-period & $\begin{array}{l}\text { Number of } \\
\text { cases }\end{array}$ & $\begin{array}{l}\text { Average annual incidence rate } \\
\left(/ 10^{5}\right)\end{array}$ \\
\hline Influenza A (H1N1) & $\begin{array}{l}2009.5- \\
2014.12\end{array}$ & 4,755 & 1.008 \\
\hline SARS & $\begin{array}{l}2005.1- \\
2014.12\end{array}$ & 1 & 0.001 \\
\hline Measles & $\begin{array}{l}2005.1- \\
2014.12\end{array}$ & 25,994 & 2.772 \\
\hline Tuberculosis & $\begin{array}{l}2005.1- \\
2014.12\end{array}$ & 34,3161 & 36.450 \\
\hline $\begin{array}{l}\text { Meningococcal } \\
\text { meningitis }\end{array}$ & $\begin{array}{l}2005.1- \\
2014.12\end{array}$ & 242 & 0.026 \\
\hline Pertussis & $\begin{array}{l}2005.1- \\
2014.12\end{array}$ & 1,606 & 0.170 \\
\hline Scarlet fever & $\begin{array}{l}2005.1- \\
2014.12\end{array}$ & 22,554 & 2.366 \\
\hline Influenza & $\begin{array}{l}2005.1- \\
2014.12\end{array}$ & 23,874 & 2.496 \\
\hline Mumps & $\begin{array}{l}2005.1- \\
2014.12\end{array}$ & 105,305 & 11.065 \\
\hline Rubella & $\begin{array}{l}2005.1- \\
2014.12\end{array}$ & 10,014 & 1.069 \\
\hline Diphtheria & $\begin{array}{l}2005.1- \\
2014.12\end{array}$ & 0 & 0 \\
\hline
\end{tabular}


Table 2

The Moran's / of global spatial autocorrelation analysis for respiratory infectious diseases in Shandong Province during the study period.

\begin{tabular}{|llll|}
\hline Diseases & Moran's I & $\boldsymbol{Z}$ & $P$-value \\
\hline Influenza A (H1N1) & 0.076 & 2.040 & 0.034 \\
\hline Measles & 0.353 & 8.664 & 0.001 \\
\hline Tuberculosis & 0.346 & 8.278 & 0.001 \\
\hline Meningococcal meningitis & 0.062 & 1.642 & 0.061 \\
\hline Pertussis & 0.238 & 6.251 & 0.001 \\
\hline Scarlet fever & 0.157 & 4.170 & 0.001 \\
\hline Influenza & 0.130 & 3.691 & 0.008 \\
\hline Mumps & 0.166 & 4.202 & 0.001 \\
\hline Rubella & 0.093 & 2.423 & 0.015 \\
\hline
\end{tabular}

Figures 

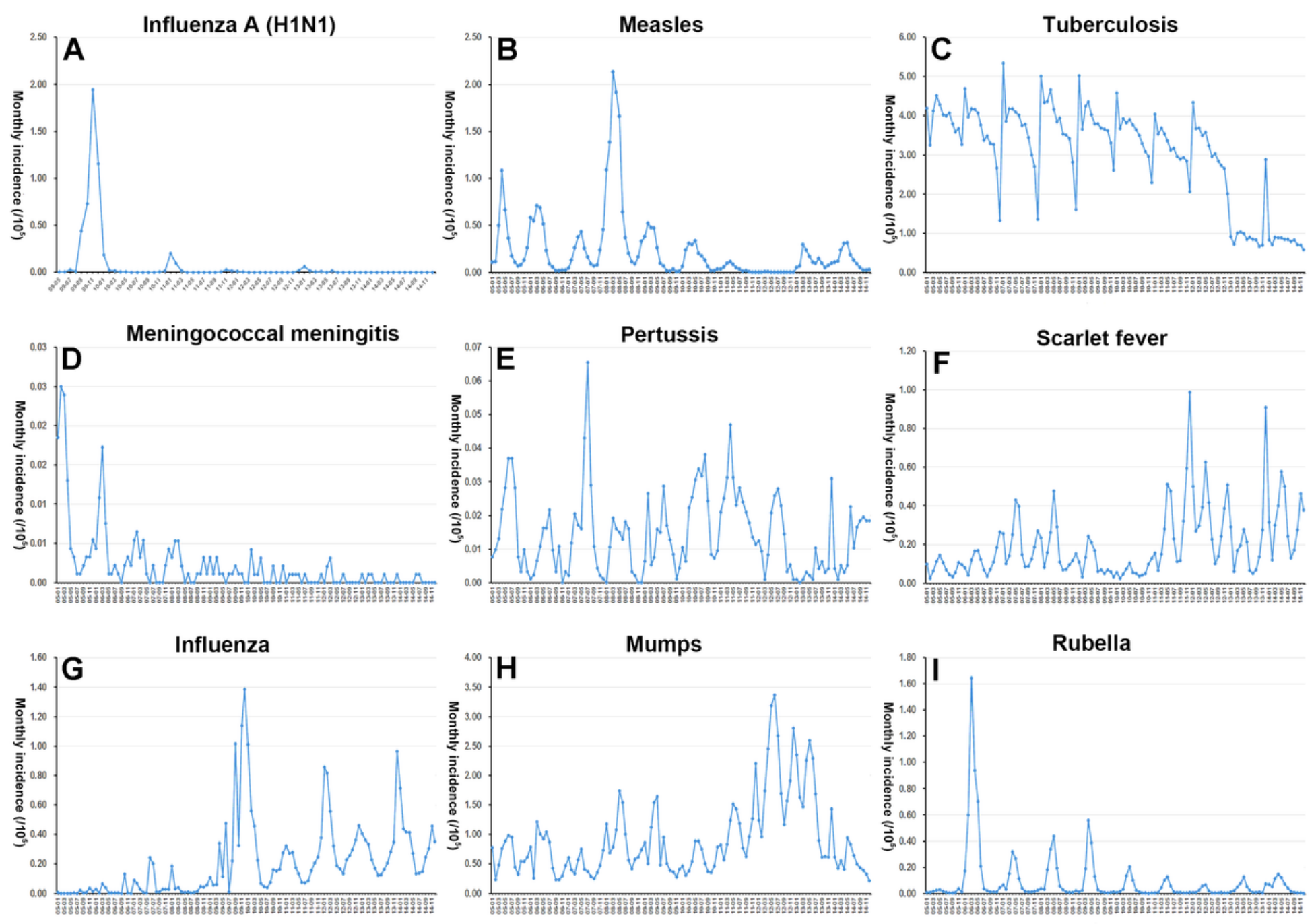

\section{Figure 1}

Monthly incidence of respiratory infectious diseases from 2005-2014 in Shandong Province. 

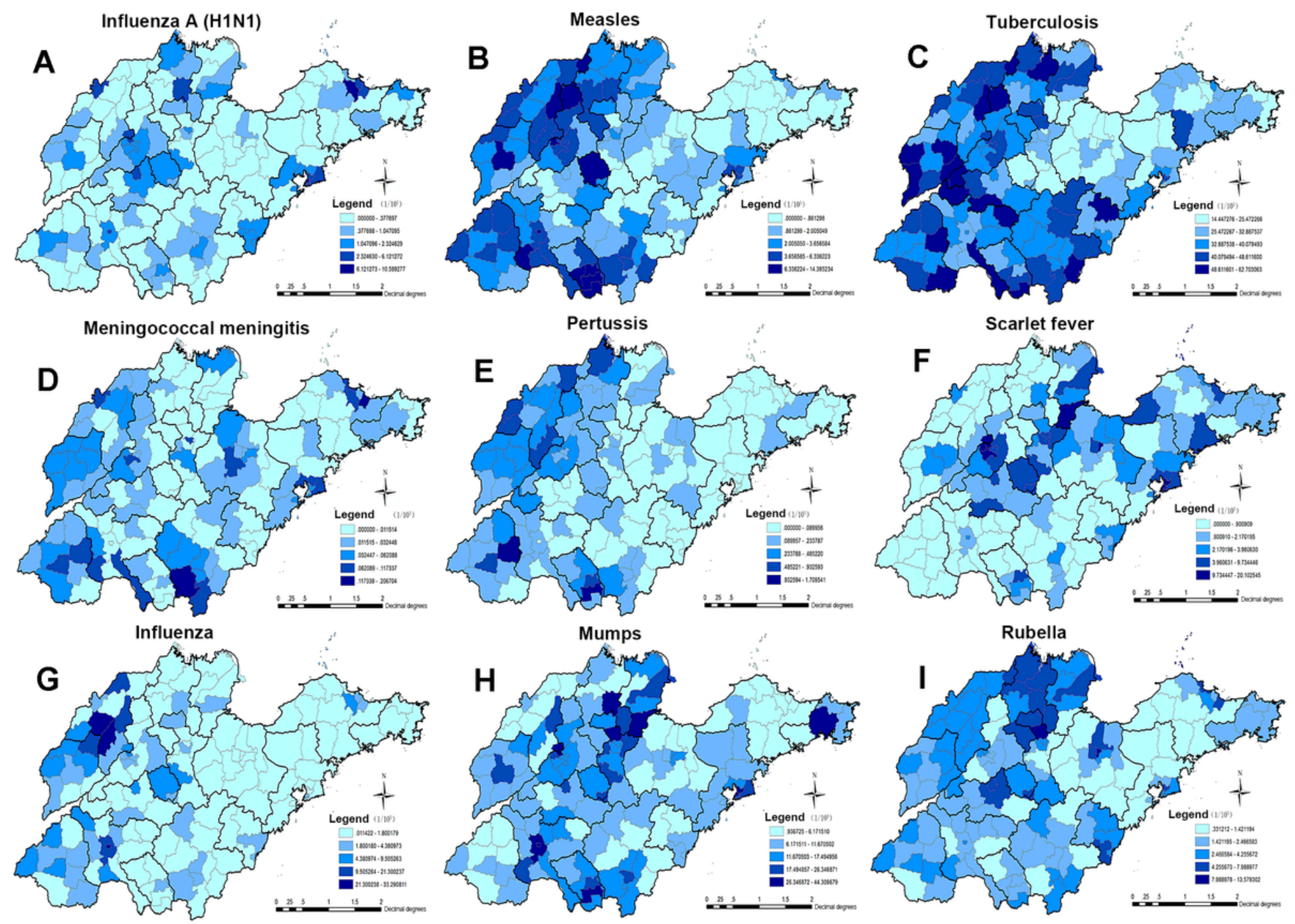

Figure 2

The average annual incidence rate of respiratory infectious diseases by the natural breaks (Jenks) method from 2005-2014 in Shandong Province. 


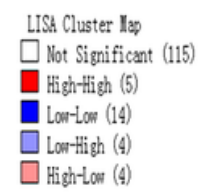

Influenza A (H1N1)

A

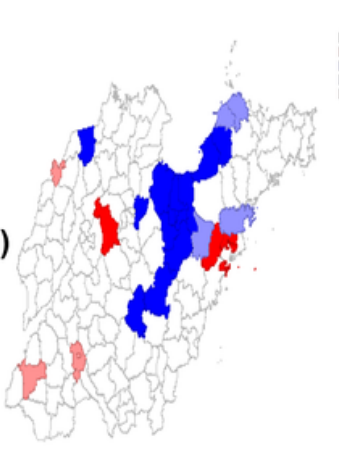

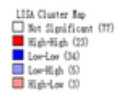
Measles

B

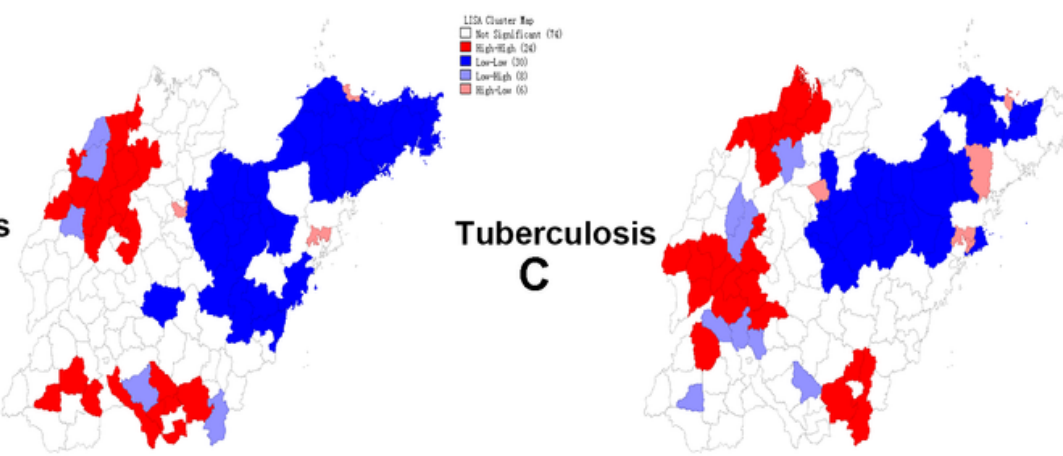

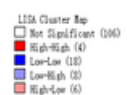

Meningococcal meningitis

D

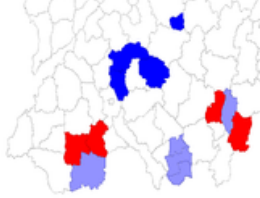

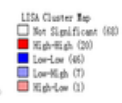

Pertussis

E

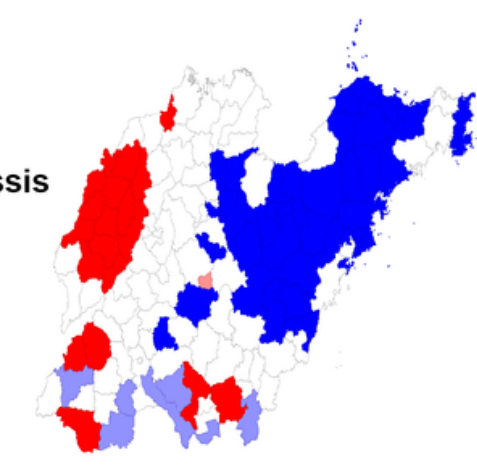

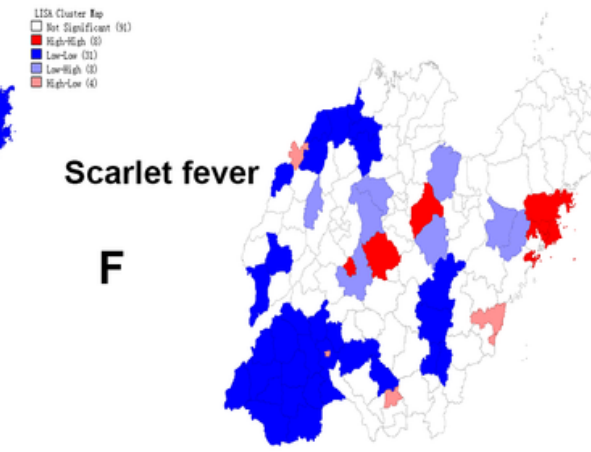

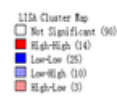

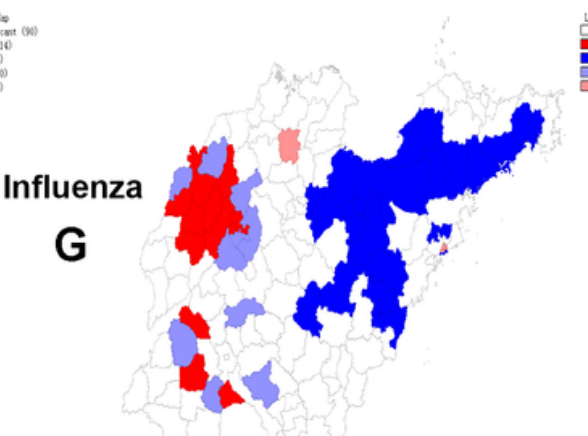

锌-

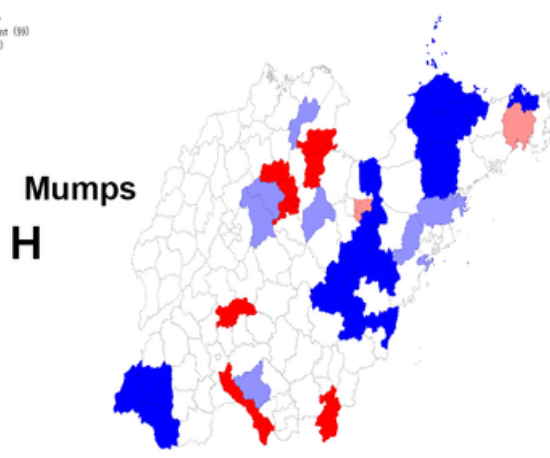

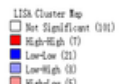

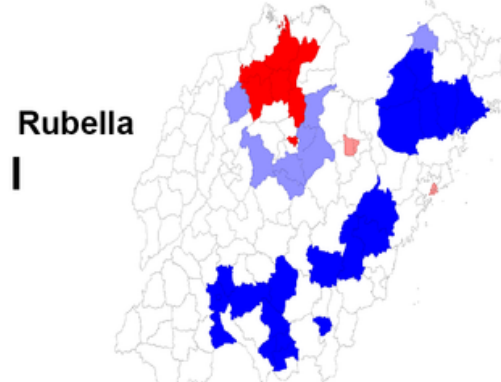

Figure 3

The LISA cluster maps of respiratory infectious diseases during 2005-2014 in Shandong Province. 
Influenza A (H1N1):

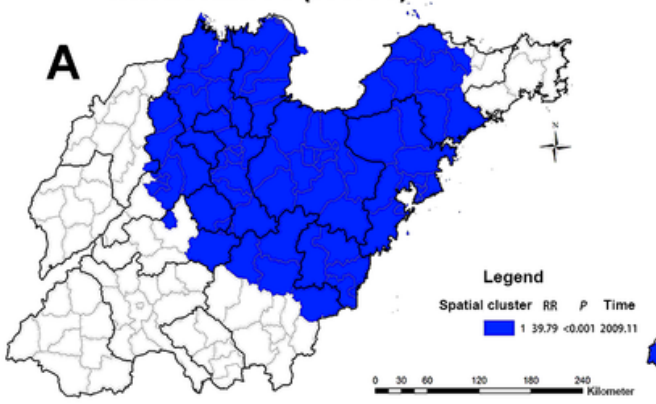

Meningococcal meningitis

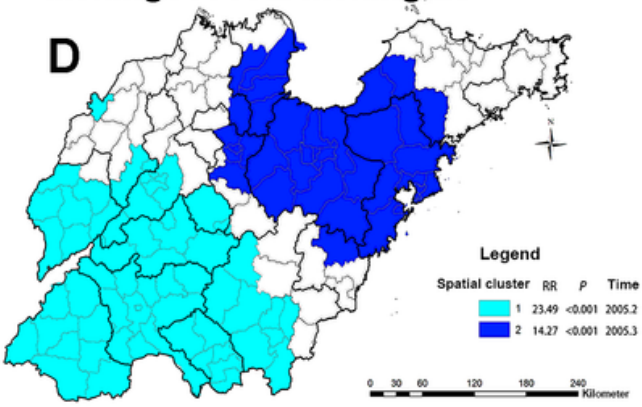

Influenza

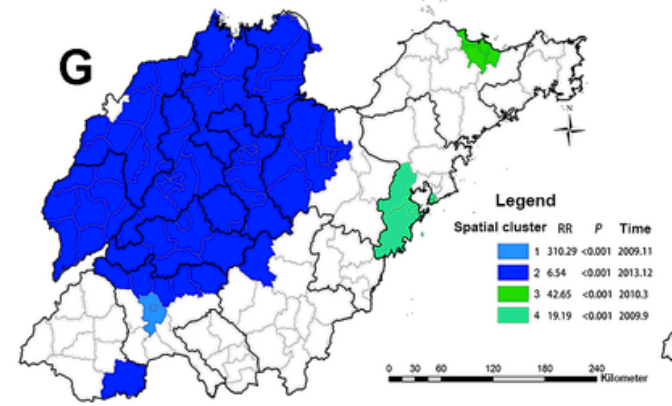

Measles

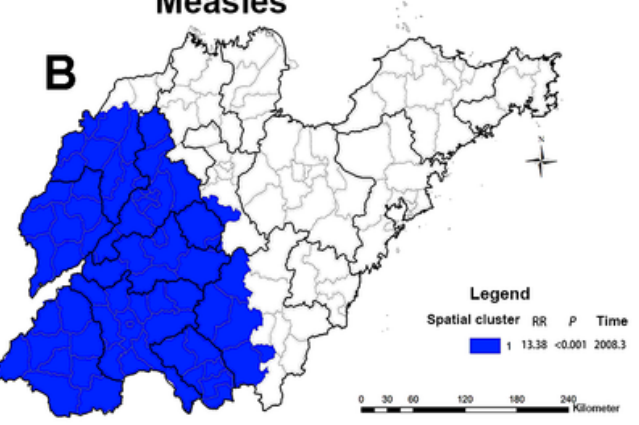

Pertussis

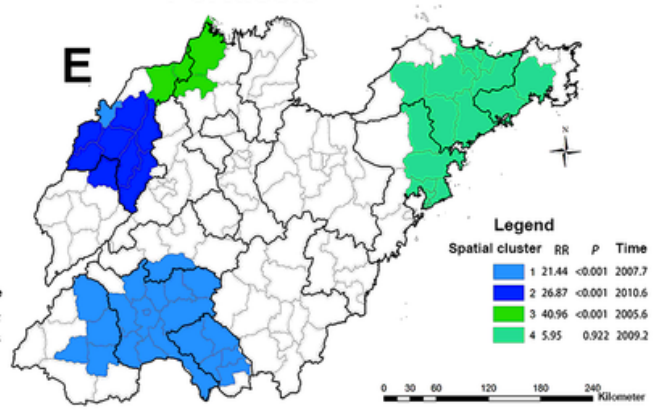

Mumps

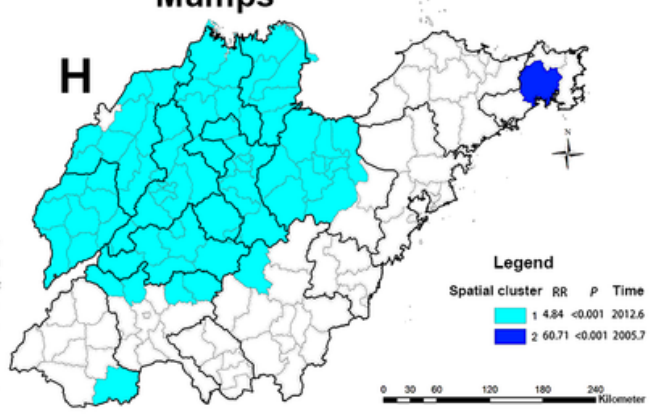

Tuberculosis

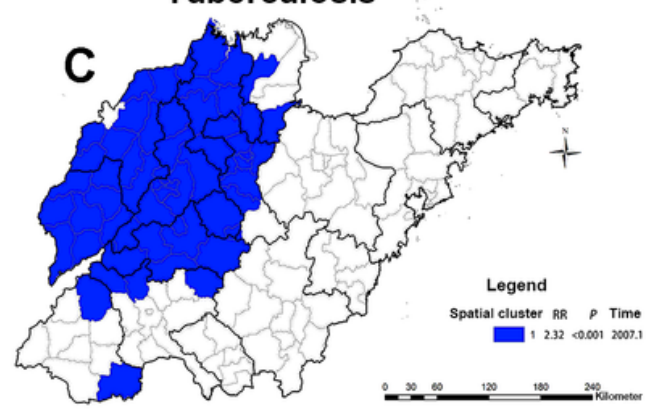

Scarlet fever

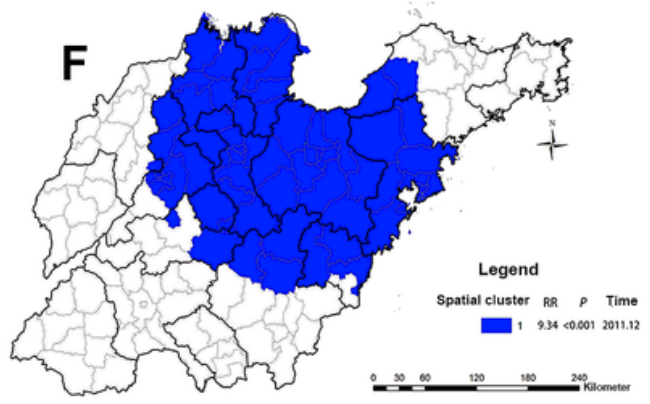

Rubella

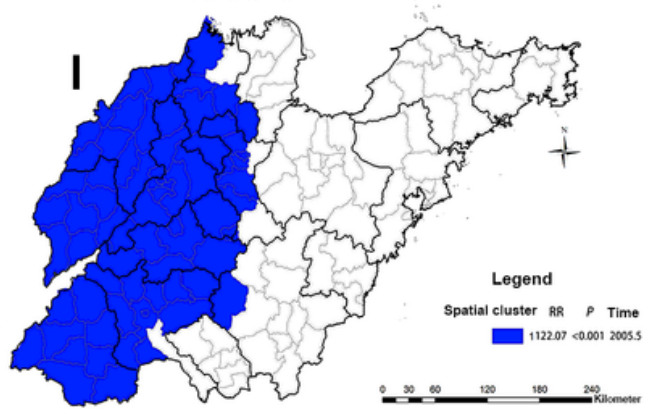

Figure 4

Spatial-temporal clusters of respiratory infectious diseases during 2005-2014 in Shandong Province.

\section{Supplementary Files}

This is a list of supplementary files associated with this preprint. Click to download.

- AdditionalFile1.docx 